\title{
Sistema SCALAWEB: busca avançada
}

\section{Roberto Franciscatto}

Professor Assistente, Universidade Federal do Rio Grande do SUl (UFRGS), Centro Interdisciplinar de Novas Tecnologias na Educação (CINTED), Av. Paulo Gama, 110 - prédio 12105 - 3- andar, sala 332. CEP. 90040-060, Porto Alegre (RS) Brasil. roberto.franciscatto@gmail.com

Recibido: 15 Fed 2016 - Revisado: 30 Abr 2016 Aceptado: 30 May 2016 - Publicado: 30 Jul 2016

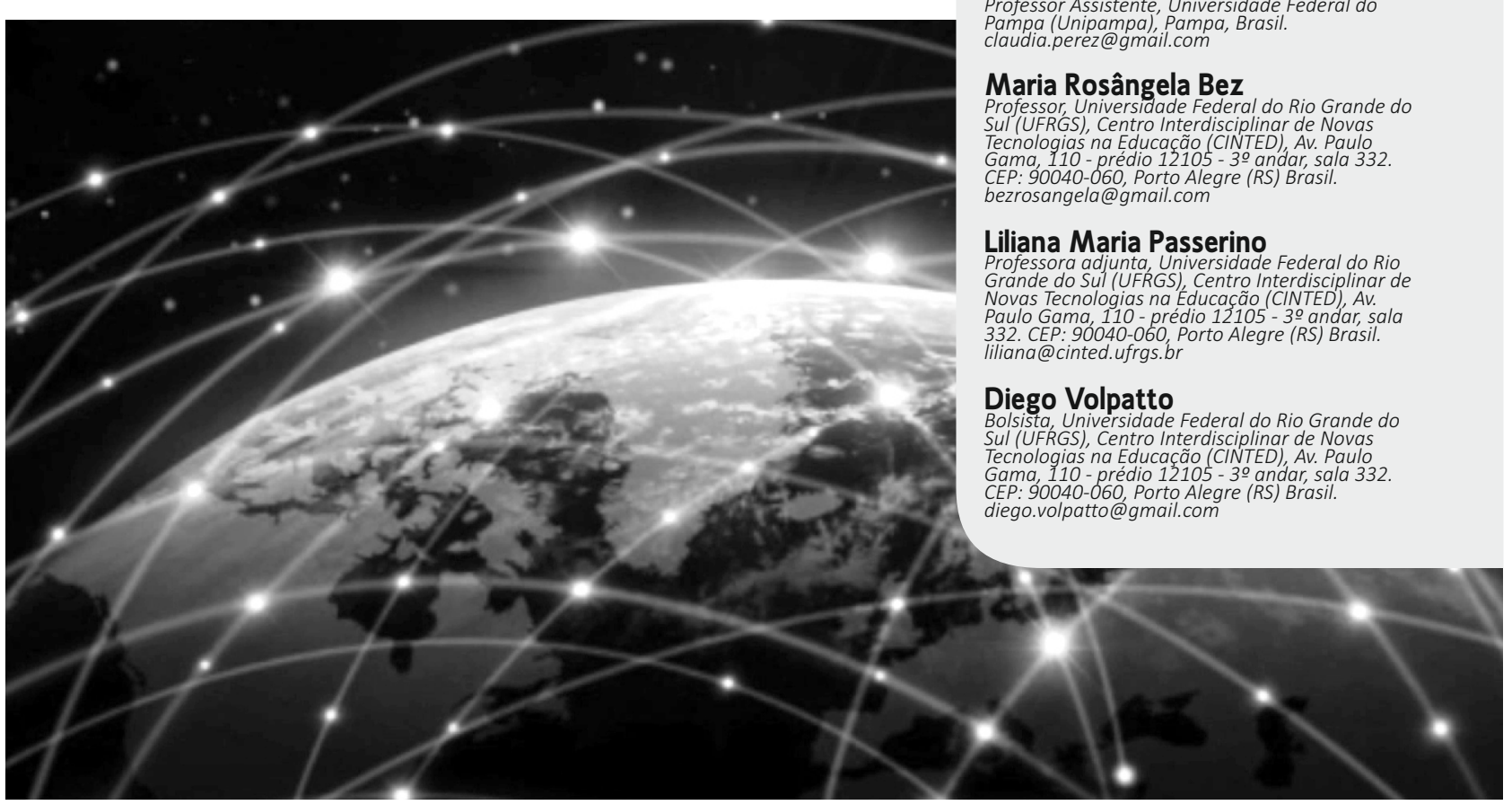

Resumo: O trabalho em questão objetiva descrever sobre o processo de desenvolvimento de um sistema de busca avançada por pictogramas, através de palavras-chave e elementos de seleção para o sistema SCALAWEB. Ao longo do texto são abordados exemplos e conceituações de tecnologias assistivas de comunicação alternativa, uma síntese sobre o sistema SCALAWEB e seus objetivos, as tecnologias e ferramentas utilizadas na implementação do sistema avançado de busca proposta para o mesmo. Ainda, são especificados o projeto, desenvolvimento e integração do sistema de busca avançada no SCALAWEB, sua forma de funcionamento, características, arquitetura e contexto de uso dentro das tecnologias assistivas, em especial ao TEA (Transtorno do Espectro Autista). Por fim, os resultados preliminares e trabalhos futuros são expostos, bem como as conclusões acerca do artigo.

Palavras-chave: busca avançada, comunicação alternativa, inclusão, SCALAWEB.

Abstract: The work in question aims to describe the process of developing of an advanced search system by pictograms, using keywords and selection elements for the SCALAWEB system. Throughout the text, examples and concepts of assistive technologies for alternative communication are discussed, a synthesis of the SCALAWEB system and its objectives and the technologies and tools used in the implementation of the proposed of this advanced search system. Moreover, the project, design, development and integration of the advanced search system in SCALAWEB are specified, how it works, characteristics, architecture and context of use within the assistive technologies, in particular to the TEA (Autism Spectrum Disorder). In the end, the preliminary results and future works are exposed, as weel as the conclusions about the article.

Keywords: advanced search, alternative communication, inclusion, SCALAWEB. 


\section{INTRODUÇÃO}

A busca de técnicas, recursos e estratégias para a autonomia de pessoas com deficiência passou a se intensificar a partir da Lei de inclusão (Brasil, Política Nacional de Educação Especial na Perspectiva da Educação Inclusiva). Somatizado a revolução tecnológica da atualidade, os recursos para este público-alvo se efetivam através das tecnologias assistivas. No caso desse trabalho, as de comunicação alternativa, através do sistema SCALAWEB - Sistema de Comunicação Alternativa para Letramento de pessoas com Autismo, composto por um recurso mais uma metodologia de uso. O sistema SCALAWEB, foi desenvolvido através de tecnologias de software livre e está disponível na web1 (UFRGS, 2015). Tem por objetivo apoiar o desenvolvimento de crianças com Transtorno do Espectro Autista na interação social e no incentivo a oralidade nos déficits de comunicação. Hoje, conta com mais de 700 usuários, desses, muitos professores que aderiram ao seu uso, após formações continuadas aos mesmos.

Durante essas formações foram realizadas avaliações do Sistema SCALAWEB e uma das solicitudes apontadas foi à falta de um sistema de busca (por palavra-chave e consequentemente avançada) que facilitasse a construção das pranchas.

Para tanto, esse trabalho inicia o estudo para que um sistema de busca avançada seja implementado no referido recurso. Assim, a composição desse artigo foi dividida em etapas. Na primeira as tecnologias assistivas de comunicação alternativa são apresentadas, como forma de situar o leitor no contexto das TAs. Em seguida, o sistema SCALAWEB é apresentado com um sucinto histórico e sua descrição de uso. As ferramentas e tecnologias utilizadas na implementação da busca avançada são descritas na sequência, seguido pela metodologia e pela detalhada descrição da arquitetura proposta para o sistema de busca avançado no SCALAWEB. Os resultados iniciais são relatados e algumas considerações finais tecidas.

\section{TECNOLOGIAS ASSISTIVAS DE COMUNICAÇÃO ALTERNATIVA}

A Comunicação Alternativa (CA) é uma das áreas da Tecnologia Assistiva, que apoia o desenvolvimento de uma comunicação mais autônoma das pessoas com déficits nesse âmbito. A CA preocupa-se com técnicas, processos e ferramentas que auxiliem a comunicação, como apoio, complementação ou substituição da fala. Seu uso justifica-se não pelo suporte midiático adotado, mas pelas estratégias e técnicas comunicativas que podem promover a autonomia dos sujeitos em situações de comunicação (Tetzchner \& Martinsen, 2000).

No caso do autismo, os déficits de comunicação podem se manifestar com alterações no uso, na forma ou no conteúdo da linguagem na pragmática e, em menor medida, no nível sintático, morfossintático, fonológico ou fonético. Desse modo, a importância de utilizar um sistema de CA está focada mais em processos de compreensão e de produção de sentidos do que em produção sonora ou morfossintática (Passerino, 2005), (Passerino \& Santarosa, 2008), (Passerino y Bez, 2013).

Estudos recentes desenvolvidos por (Avila, 2011) e (Avila \& Passerino, 2011), envolvendo o uso de CA com sujeitos com Transtorno do Espectro Autista, apresentam resultados importantes. Esses resultados são significativos, especialmente, quando se apoia os processos de CA no uso de tecnologias digitais para o desenvolvimento da comunicação e da interação social de sujeitos com autismo.

Softwares de CA têm sido desenvolvidos para desktops, como por exemplo, Boardmaker, Plaphoons, E-triloquist, Amplisoft, dentre outros, ou para uso na web, de forma online como: Askability, Symbolworld, PICTO4ME, para uso direto com o usuário ou utilizados na elaboração de atividades ou pranchas de comunicação. Assim como para uso em dispositivos móveis, como por exemplo, AraBoard, 
Grid Player, My Voice My Words, PictoDriod Life, Dílo, dentre outros. Observa-se que a grande maioria desses recursos ou são pagos ou não foram desenvolvidos pensando-se no público do TEA (Transtorno do Espectro Autista) que estejam em fase inicial de letramento. Dentro desta perspectiva surge o SCALAWEB, que se apresenta na sequência desse trabalho.

\section{SISTEMA SCALAWEB}

No ano de 2009, iniciou-se o desenvolvimento do sistema SCALAWEB com o intuito de ser projetado um software de comunicação alternativa que incluía não somente um programa de computador, mas uma metodologia de uso para apoiar o processo de desenvolvimento da linguagem de crianças com TEA (Transtorno do Espectro Autista) que apresentassem déficits na comunicação oral. Focado numa epistemologia sócio-histórica, tanto na concepção como no desenvolvimento e aplicação do mesmo, isso implica numa reorganização conceitual do processo de desenvolvimento de software conhecido como Design Centrado no Usuário (DCU) para um Desenvolvimento Centrado em Contextos de Uso (DCC) que ultrapassa a análise somente da interação sujeito-objeto e foca em processos de interação sujeito-objeto-sujeito, na qual o objeto se estabelece como instrumento de mediação (Bez \& Passerino, 2009), (Bez, 2010) (Bez, 2014).

O sistema SCALAWEB possui um programa de computador composto por três módulos: prancha, narrativas virtuais e comunicação livre. No módulo prancha é possível construir pranchas de comunicação. No módulo narrativas visuais pode-se preparar histórias e no módulo de comunicação livre se dá á conversação através de um chat. Além de funcionalidades comuns entre os aplicativos tais como: importar imagens, editar sons, salvar, exportar e gerenciar os diferentes arquivos gerados pelo sistema, cada módulo possui funcionalidades específicas. O menu à esquerda apresenta ao usuário as categorias de imagens que podem ser utilizadas em todos os módulos enquanto a barra horizontal de menu apresenta suas funcionalidades.

Para a construção de pranchas no SCALA, elege-se um layout (cinco opções) e, após, adiciona-se o(s) pictograma(s). Na tela principal do sistema, lado esquerdo, encontram-se as oito categorias de pictogramas denominadas/divididas por: Pessoas, Objetos, Natureza, Ações, Alimentos, Sentimentos, Qualidades e Minhas Imagens. As categorias possuem pictogramas relacionados com a sua denominação. Por exemplo, a categoria «Pessoas» possui pictogramas de familiares, profissões, nacionalidades; a categoria «Objetos» imagem de bola, caixa, pia, etc. Na categoria «Minhas Imagens», há a possibilidade do usuário importar imagens de sua preferência ou de acervo pessoal para o SCALAWEB como, por exemplo, importar imagens dos membros da família, imagens de alunos de determinada turma, etc. Para inserir uma imagem na prancha é preciso clicar em uma categoria e selecionar a imagem desejada, após indicar o lugar de destino na prancha. A Figura 1 ilustra duas imagens da categoria «Ações», respectivamente «Abrir a torneira» e "Lavar as mãos».

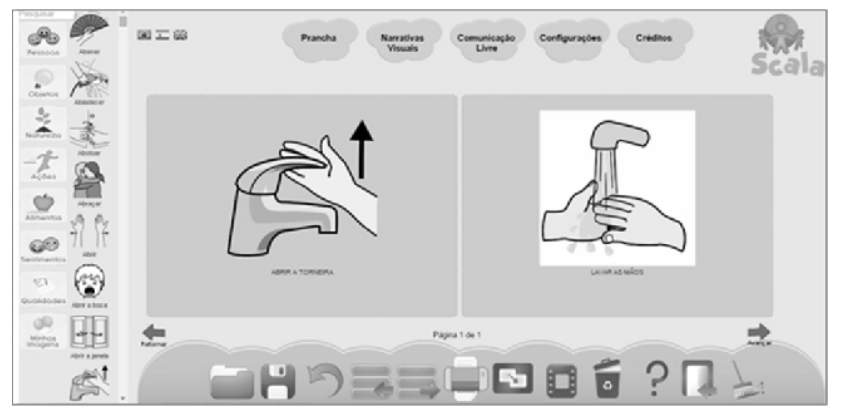

Figura 1. Função Inserir imagem.

No processo de importação de uma imagem é necessário que o usuário clique na função importar e selecione a imagem desejada no(s) diretório(s) do computador pessoal. As imagens importadas ficam na categoria «Minhas Imagens». 
Avaliando o conjunto de funções do SCALA sobre o processo de construção de uma prancha, observa-se que aprimoramentos no sistema podem ser realizados, como é o caso dos processos de inserção e importação de imagem, que exige muitos passos e não contam com uma ferramenta de busca mais automática, tornando o processo mais lento e dificultando a ação de um usuário com ou sem experiência.

Uma alternativa é incluir no sistema a busca avançada que em geral considera vários pontos, incluindo contexto de pesquisa, a localização, a intenção, a variação das palavras, tratamento de sinônimos, consultas generalizadas e especializadas, conceito de correspondência e consultas em linguagem natural para fornecer resultados de pesquisa relevantes.

Com a busca avançada de imagens, resultados mais automáticos e relevantes podem ser disponibilizados, eliminando um conjunto de passos dos processos de inserção e importação de imagem (ns) no sistema. Para a proposição de uma solução de busca avançada no SCALAWEB é necessário a retomada dos requisitos do sistema e de sua modelagem, para o desenvolvimento de funcionalidades que atuem na busca automática de imagem.

\section{FERRAMENTAS E TECNOLOGIAS UTILIZADAS NA IMPLEMENTAÇÃO DO SCALAWEB}

O desenvolvimento do SCALAWEB envolve uma série de tecnologias e ferramentas que permitem a integração de serviços e pleno funcionamento das funcionalidades projetadas para o mesmo. Nas seções subsequentes são apresentadas as principais ferramentas e tecnologias como forma de entendimento ao capítulo de desenvolvimento e funcionamento da arquitetura do SCALAWEB.

\subsection{HTML, CSS, AJAX, PHP e PostgreSQL}

Para construção das interfaces de comunicação com o usuário, também conhecidas como front-end, foi utilizada a linguagem de estruturação/marcação HTML e as folhas de estilo CSS. A linguagem HTML é utilizada para a construção de páginas/sites web que são interpretadas por navegadores (browsers). Já as folhas de estilo em CSS permitem ao desenvolvedor alterar a aparência dos documentos escritos em linguagem de estruturação/marcação como o HTML. O AJAX (JavaScript e XML assíncrono, tradução livre) é uma tecnologia que permite a troca de informações de forma assíncrona entre a aplicação SCALAWEB e o banco de dados, por exemplo, como forma de prover mais interatividade ao usuário e a resposta esperada pelo mesmo (usado este recurso no sistema de busca por palavra-chave no SCALAWEB).

A linguagem de programação PHP, também conhecida como server-side, é uma linguagem interpretada, utilizada para construção e conteúdo dinâmico em sites. O código escrito é interpretado pela linguagem PHP (sendo necessário para isso, o servidor PHP devidamente configurado), que gera as páginas web visualizadas no lado do cliente (Praciano, 2014). Esta linguagem foi utilizada na programação do conteúdo back-end do SCALAWEB, juntamente com o HTML e as folhas de estilos CSS, descritas no parágrafo anterior. Por fim, como sistema gerenciador de banco de dados escolhido para o SCALAWEB optou-se pelo PostgreSQL. Este é um gerenciador de banco de dados de código aberto, avançado, que conta com recursos como: consultas complexas, facilidade de acesso, gatilhos, integridade transacional, entre outros e flexibilidade quanto as rotinas básicas de criação de tabelas, consultas, edição e atualizações. O modelo Entidade-Relacionamento (ER) projetado para a base de dados do SCALAWEB é apresentada na seção de desenvolvimento.

\subsection{Ontologias e Protégé}

Ontologia é a descrição de categorias de "coisas» que existem ou podem existir em um determinado domínio de conhecimento. As ontologias têm sua estrutura baseada na descrição de conceitos e dos 
relacionamentos semânticos entre eles. A ontologia é uma definição formal e explícita dos conceitos (classes ou categorias) compartilhados, presentes num domínio, bem como, de seus atributos, propriedades e relações (Berners-Lee, Hendler \& Lassila, 2011) (Noy \& Mcguinness, 2001). Logo uma ontologia fornece um vocabulário que descreve um domínio de uma determinada área do conhecimento, sendo que estes vocabulários por vezes podem ser especificados de diferentes maneiras. As linguagens utilizadas na especificação de ontologias podem ser agrupadas em três tipos (Almeida \& Bax, 2003): linguagens de ontologias tradicionais (Cycl, Ontolíngua, F-Logic, CML, OCML, Loom, KIF), linguagens padrão Web (XML, RDF) e linguagens de ontologias baseadas na Web (OIL, DAML+OIL, SHOE, XOL, OWL). Para a criação das ontologias no sistema SCALAWEB, foram definidas as categorias do próprio sistema tais como: Pessoas, Objeto, Natureza, Ações, Alimentos, Sentimentos, Qualidades e Minhas Imagens.

Para que esta descrição/definição das ontologias fosse projetada, construída e organizada, foi utilizada a ferramenta Protégé (http://protege.stanford.edu/). O Protégé é uma ferramenta livre, de código aberto, que possui um conjunto de ferramentas para a construção de modelos de domínio e aplicações baseadas no conhecimento de ontologias. Na Figura 2, é possível visualizar a estrutura inicial implementada para o SCALAWEB quanto a busca semântica pretendida para o mesmo.

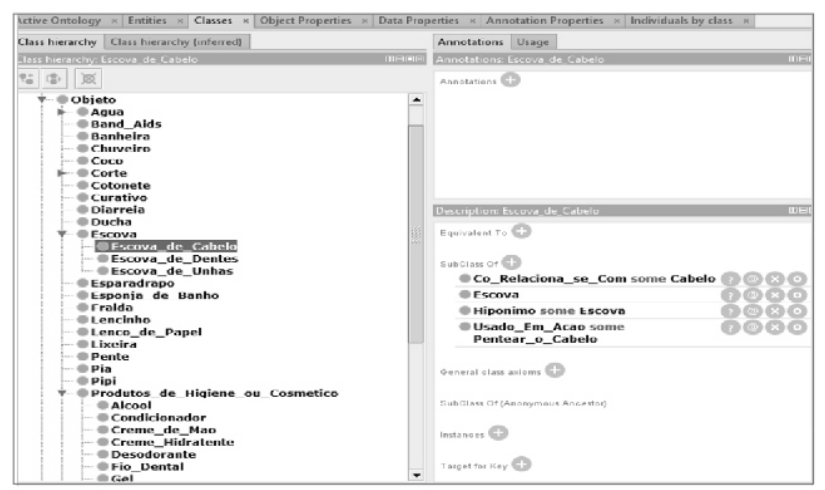

Figura 2. Implementando Classes no Protégé.
No ambiente de construção de ontologias Protégé foram utilizadas as Classes e Object Properties. As Classes são a representação concreta de um conceito ou entidade, interpretadas também como conjuntos que podem conter indivíduos da ontologia e Object Properties são as relações binárias entre indivíduos, ou seja, relaciona um indivíduo a outro indivíduo.

Para a ontologia projetada no Protégé, um cenário de uso inicialmente pensado para o SCALAWEB foi a «Higiene pessoal» de crianças entre 4 e 7 anos, para responder questões norteadores do tipo: «Como uma prancha de comunicação pode auxiliar crianças entre 4 e 7 anos na higiene pessoal?», «Como uma prancha de comunicação pode auxiliar a criança na escovação de dentes?», "Como uma prancha de comunicação pode apresentar a ação de tomar banho e os objetos que estão envolvidos no processo», entre outras.

Logo, os conjuntos de classes definidas para este cenário foram: Ação, Local, Objeto, Partes do Corpo e Pessoa. Conforme a necessidade, também foram criadas Subclasses, ou seja, subconjunto dentro das classes, como por exemplo, a Classe Escova e as Subclasses Escova de Cabelo, de Dentes e de Unhas. As classes e subclasses estão associadas aos pictogramas do banco de imagens do SCALAWEB.

As Object Properties da ontologia foram projetadas com base nos relações e respectivas descrições definidas para as ontologias WordNet (http://www.clul.ul.pt/wn/) e Papel (http://www.linguateca.pt/PAPEL/), resultando no conjunto que segue: Antônimo de (Papel), Sinônimo de (WordNet), Finalidade de (Papel), Usado em Ação (Papel), Parte de (WordNet), Tem como Parte (WordNet), Correlaciona-se com (WordNet), Hipônimo (WorNet) e Lugar para (WordNet). As propriedades "Sinônimo», "Finalidade de» e «Parte de» possuem propriedades inversas correspondentes a «Antônimo de», «Usado em Ação» e «Tem como Parte». Para as relações da ontologia foram utilizadas as propriedades Annotation (Comment) e Description 
(Inverse of) usadas para vincular metadados as Object Properties, conforme figura 3.

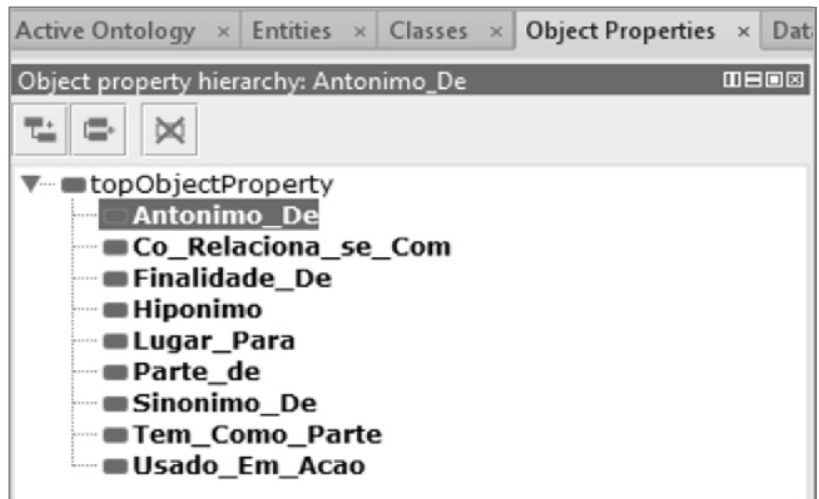

Figura 3. Object Properties - Protégé.

Como os indivíduos da ontologia são pictogramas, as relações também foram marcadas com as características Functional e Symmetric. Uma propriedade é dita funcional para dado indivíduo, quando ele pode se relacionar a apenas um outro indivíduo a partir da relação. Já uma relação $\mathrm{R}$ binária é simétrica se para qualquer indivíduo $a$ e $b, a R b$ implica em bRa.

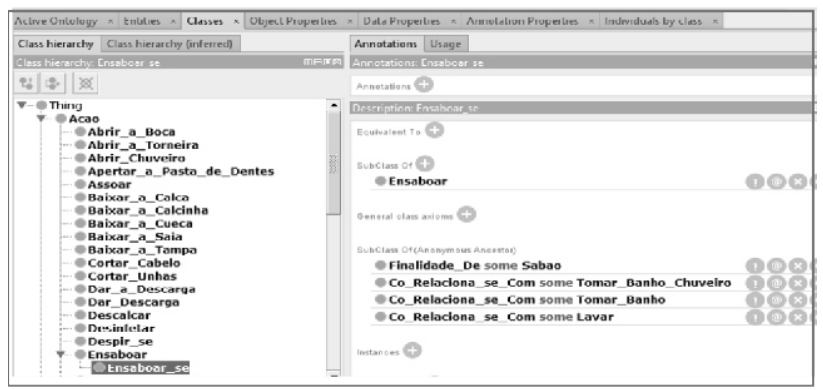

Figura 4. Classes, SubClass Of e SubClass Of (Anonymous Ancestor) - Protégé.

Automaticamente o Protégé defini propriedades entre indivíduos, com a descrição SubClass of (Anonymous Ancestor). O trabalho resultou a ontologia com um valor de Classes (208), Object Property (9), SubClass Of - relações entre as classes (974) e SubClass Of (Anonymous Ancestor) (716).

\subsection{Apache Jena e Sparql}

O Apache Jena é um framework desenvolvido na linguagem Java, para construção de aplicações de Web Semântica. Inclui um motor de inferências baseado em regras, além de fornecer um ambiente de banco de dados através do SPARQL. O Apache Jena (servidor) é utilizado junto ao SCALAWEB como forma de prover a infraestrutura necessária ao contexto semântico que o mesmo necessita na busca avançada, possuindo uma interface gráfica de gerenciamento, consultas e visualização.

Já o SPARQL, constitui-se como um subsistema de banco de dados para o Apache Jena. Possui recursos como balanceamento de carga, segurança, clustering, backup e administração. No SCALAWEB permite realizar as consultas das tuplas geradas em formato OWL, exportados através da ferramenta Protégé, extraindo os dados e disponibilizando-os no formato JSON, ficando disponíveis através do Apache Jena a API doSCALAWEB.

\subsection{JSON e APIS}

O JSON é um modelo para armazenamento e transmissão de informações no formato texto. Sua utilização em aplicações web dá-se principalmente pela capacidade de estruturar informações de uma forma mais compacta do que o formato $\mathrm{XML}$, por exemplo (Devmedia, 2014). No SCALAWEB tem um papel importante que é preparar os dados que serão utilizados pela API, como retorno das consultas originárias pelo SPARQL.

Já uma API web, nada mais é do que um conjunto de rotinas e padrões de programação para acesso a um aplicativo ou plataforma baseada na web. No caso do sistema SCALAWEB, a ideia é a implementação de uma API de transmissão (quando a pesquisa necessita enviar dados ao SPARQL) e uma de recepção (quando necessita retornar a aplicação SCALAWEB o resultado das relações existentes nas ontologias pesquisadas). 
As ferramentas e tecnologias apresentadas nesta seção são o aporte necessário à implementação e integração dos recursos e serviços necessários e fundamentais ao desenvolvimento da busca avançada no SCALAWEB. Sua perfeita integração e testes são vitais para o sucesso do projeto, bem como, atualizações e melhorias no mesmo.

\section{METODOLOGIA}

Esse artigo tem por objetivo a implementação de um sistema de busca avançada/semântica para o sistema SCALAWEB. Efetivando-se numa pesquisa qualitativa conceituada por (Godoy, 1995) pela «obtenção de dados descritivos sobre pessoas, lugares e processos interativos pelo contato direto do pesquisador com a situação estudada, procurando compreender os fenômenos segundo a perspectiva [...]», dos participantes da situação. Nesse trabalho pelos estudos acerca da busca semântica através de ontologias com posteriores construções de relações e mapeamento de um determinado contexto de uso. Esse contexto visto e analisado numa visão sóciohistórica, onde o recurso SCALAWEB foi pensado não como mero instrumento, mas como mediador de desenvolvimento enquanto instrumentos psicológicos. Num modelo social da deficiência que entende o recurso não como elemento isolado, mas inerentemente inserido e intrincado em processos sociais de produção (Walter, 2011).

\section{BUSCA AVANÇADA NO SCALAWEB - PROJETO, DESENVOLVIMENTO E INTEGRAÇÃO}

O Sistema SCALAWEB em sua essência permite a busca por pictogramas, baseado na seleção manual (clique e escolha) de imagens que estão dispostas em sete categorias principais: pessoas, objetos, natureza, ações, alimentos, sentimentos e qualidades. O grande problema deste método de escolha de pictogramas é que com o aumento da quantidade de pictogramas (cerca 4.000 imagens que compõe o banco local) disponível ao usuário a seleção manual tornou-se um processo cada vez mais trabalhoso devido ao tempo gasto na procura de um pictograma.

Baseado no problema apresentado, um sistema de busca por palavra-chave foi inicialmente projetado, como forma de trazer rapidez na busca por pictogramas e melhorar a experiência do usuário ao utilizar o sistema. Nesta busca simplificada, optou-se por utilizar apenas dados já armazenados no banco de dados para melhorar a forma como os pictogramas são procurados pelos usuários. A figura 5 demonstra o modelo Entidade-Relacionamento (ER) da base de dados que compõe o sistema SCALAWEB.

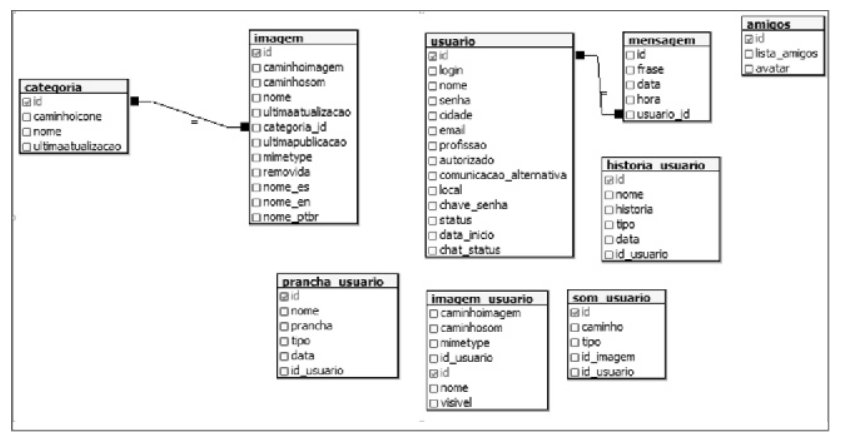

Figura 5. Modelo ER da Base de dados existente no sistema SCALAWEB.

A grande maioria dessas tabelas apresentadas faz parte da gerencia de usuários e possui características que melhoram a experiência do mesmo ao utilizar o sistema de forma que um usuário possa ter suas próprias imagens e sons, mandar mensagens para amigos dentro do próprio sistema e criar, por exemplo, uma nova prancha ou história, podendo salvá-la de modo público ou privado.

Outras tabelas vitais ao sistema são as denominadas imagem e categoria. Na tabela imagem, como o próprio nome sugere, são inseridos todos os registros de todas as imagens que o sistema SCALAWEB possui disponível ao usuário. Para isso, essa tabela possui atributos como o caminho onde estão guardadas as mídias pertinentes a esse registro, os devidos nomes 
dado as imagens em suas respectivas línguas, bem como, meta dados de inserção e atualização do mesmo. Além disso, ainda na tabela imagem, é possível visualizar um atributo identificador de categoria que remete à tabela de mesmo nome. Essa tabela é responsável por armazenar o nome da categoria, a localização do ícone que deve ser apresentado ao usuário, bem como, metadados de atualização do registro na tabela.

Para implementar a busca por pictogramas através da inserção de palavras-chave, foi adicionado um campo de entrada de dados do tipo texto com o nome de «pesquisa». Este campo foi colocado logo acima dos botões verticais de seleção de categorias, de forma não intrusiva e mantendo o mesmo padrão e estilo dos mesmos. Um exemplo desta busca, pode ser visualizada na figura 6 .

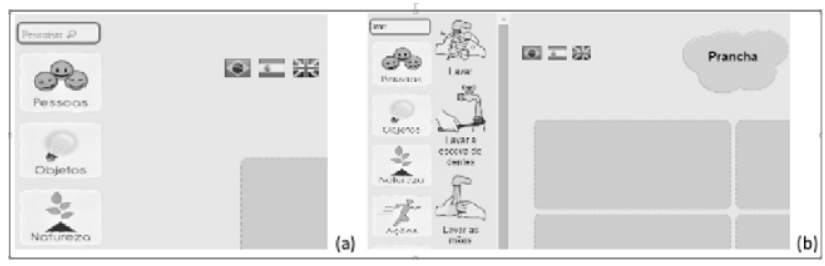

Figura 6. Botão Pesquisa (a) e pesquisa com a palavra «lavar»(b).

Após a implementação dessa solução o usuário é capaz de procurar por palavras-chave que estão relacionadas ao nome, nos idiomas português, espanhol ou inglês dos respectivos pictogramas. A exemplo da figura 6 , onde o usuário pesquisa pela palavra «lavar» e é capaz de obter uma resposta rápida, com todos os pictogramas cadastrados na base relacionados à palavra pesquisada.

Uma vez implementada a busca por palavras-chave, surgiu a necessidade de projetar algo no sistema que aumentasse o poder da busca, aumentando as opções e trazendo mais recursos aos usuários em uma busca denominada de «busca avançada». A partir de então, a implementação da busca avançada no SCALAWEB foi projetada, permitindo a seleção de alguns campos iniciais, conforme pode ser visualizado na figura 7

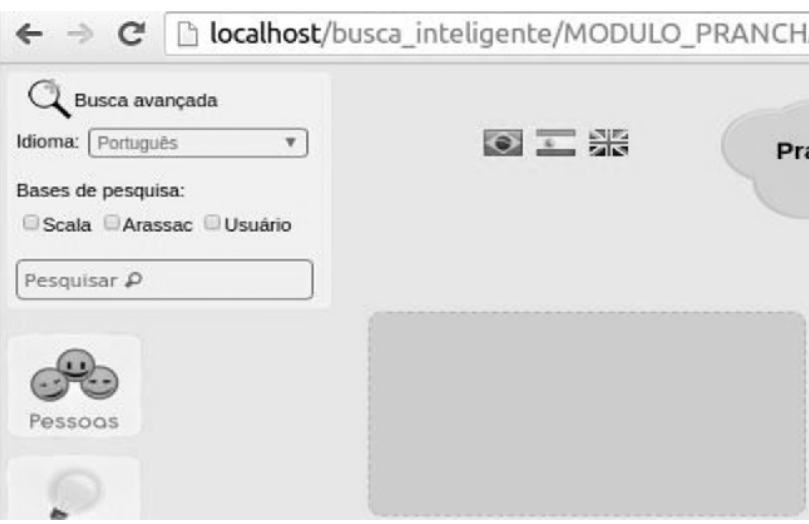

Figura 7. Front-end da busca avançada e as opções de seleção.

Estes campos são a seleção do idioma a ser efetuada a busca (disponível em português, espanhol e inglês) e as bases de dados onde será feita a pesquisa de pictogramas, podendo o usuário selecionar a base local do Scala e/ou a base externa do sistema Arasaac (http://arasaac.org/) e/ou a base personalizada de imagens salvas pelo próprio usuário (pictogramas onde o mesmo fez o upload para dentro do sistema SCALAWEB e nomeou os arquivos). Uma vez selecionados os campos de escolha, o usuário digita o nome (ou parte dele, sendo que a partir do quarto caracter inicia-se a busca) do pictograma que deseja realizar a pesquisa. A partir deste procedimento, entre em cena a arquitetura de busca avançada projetada, conforme pode ser visualizada na figura 8.

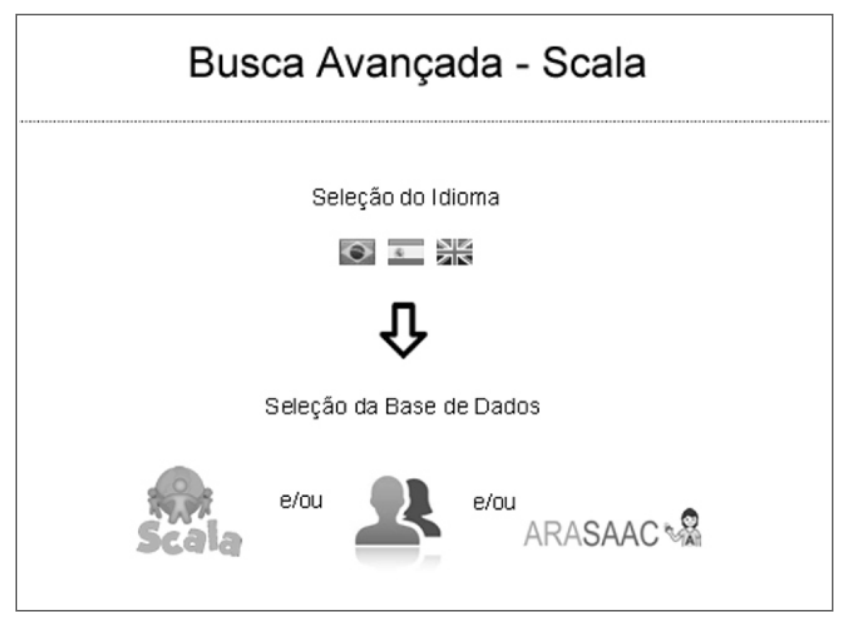




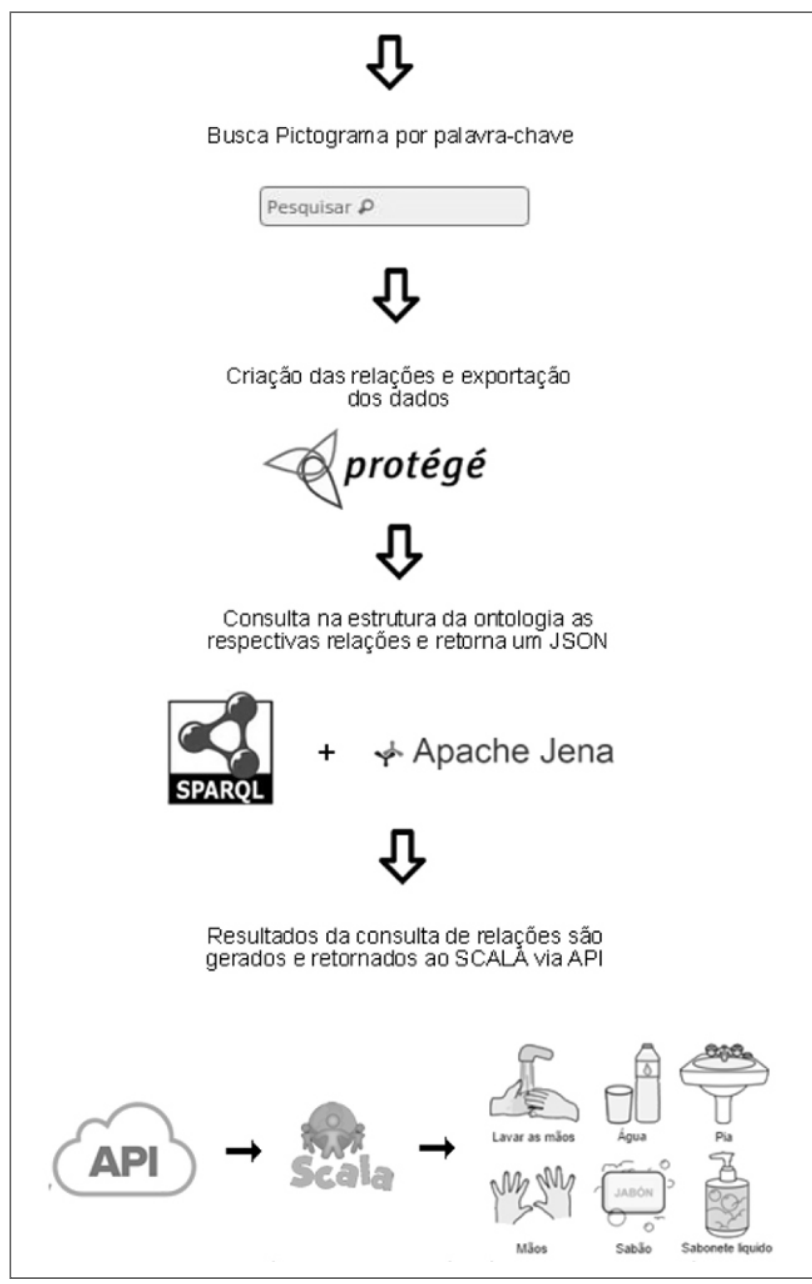

Figura 8. Arquitetura do sistema de busca avançada para o sistema SCALAWEB.

Em uma estrutura semântica secundária, implementada primeiramente através do software Protégé, as ontologias são exportadas, contendo de forma geral as relações existentes entre um pictograma e o contexto em que este possa estar inserido. Devido à complexidade desta operação, alguns estudos específicos foram desenvolvidos como forma de estabelecer tais relações e verificar sua efetiva prática com a realidade do sistema. Em um segundo momento estas ontologias são interpretadas em um servidor contendo o Apache Jena em conjunto com o banco de dados Sparql, relacionando o pictograma buscado pelo usuário e suas respectivas relações encontradas, baseadas nas ontologias descritas. Estes dados de respostas são retornados em um formato de dados JSON. A partir de então, são disponibilizados no servidor Apache Jena, para que possam ser lidos por uma API web, que fará a comunicação com a sistema SCALAWEB retornando os pictogramas correspondentes.

Como resultado desta busca avançada, uma vez que o usuário digitou uma palavra-chave correspondente e um conjunto de ontologias foi verificado e retornado via $\mathrm{API}$ as relações existentes, os resultados são apresentados no SCALAWEB através de pictogramas. Conforme exemplo da figura 8 , a palavra pesquisada "lavar» retornou ao final do processamento os pictogramas: lavar as mãos, água, pia, mãos, sabão e sabonete líquido, o que facilita para o usuário as opções de utilização de pictogramas, sem que seja necessária uma nova pesquisa para encontrar pictogramas relacionados ao buscado. Além disso, a busca avançada permite aumentar as possibilidades de utilização do sistema, tanto na construção de pranchas de comunicação, quanto de narrativas visuais (recurso disponível para ambos).

\section{RESULTADOS PRELIMINARES E TRABALHOS FUTUROS}

Como resultados preliminares acerca do protótipo de busca para sistema web SCALA, o mesmo tem sido utilizado de forma experimental entre os cerca de 700 usuários do sistema. O banco de dados de imagens recebeu atenção especial no que diz respeito a não duplicação de imagens, bem como, revisão dos nomes das imagens em suas respectivas línguas (português, espanhol e inglês). Verificações específicas nos campos da tabela imagem, como: «id», "caminhoimagem», "categoria id» e «removida», foram revisadas em mais de 4000 registros presentes no banco, como forma de garantir que o nome (número- id) dos pictogramas estivesse referenciado 
no caminho correto de gravação dos mesmos junto à plataforma SCALAWEB. Da mesma forma, «categoria_id» deve estar devidamente relacionada com o pictograma em questão, para que este se enquadre em uma categoria que tenha relação a si mesmo. Por fim, o campo «removida» passou por revisões quanto aos pictogramas que ainda encontram-se disponíveis no sistema ou que não fazem mais parte do mesmo, sendo assim um campo do tipo boolean (true ou false).

Ainda quanto a seleção de diferentes bases de dados para a busca de pictogramas, experimentos iniciais, dão conta de forma satisfatória do que diz respeito as variáveis: tempo de resposta, performance e experiência do usuário na seleção das bases locais SCALA e USUÁRIO. A busca na base de dados do ARASAAC (externo via API disponível pela própria plataforma) internamente pelo sistema SCALAWEB também funcionou nos experimentos de forma plenamente satisfatória, aumentando de forma considerável a quantidade de pictogramas e relações possíveis a serem buscadas localmente na busca avançada. No que diz respeito a arquitetura geral apresentada na figura 8, projetada e descrita neste trabalho, pretende-se reduzir o número de etapas, bem como, disponibilizá-la para testes aos usuários do sistema, como forma de validar as relações encontradas e implementar novos mecanismos como o aprendizado automático de relações propostas pelo usuário e armazenamento local.

\section{CONCLUSÕES}

Este trabalho apresentou um sistema de comunicação alternativa para letramento de crianças com autismo, denominado SCALAWEB, bem como, os recursos que o mesmo dispõe. Neste trabalho em específico, o sistema de busca avançada por palavra-chave, foi descrito, assim como, o processo de criação, implementação do mesmo e sua forma de funcionamento.

Fica notório a importância de tais sistemas, frente à quantidade de pessoas com necessidades especiais e a relevância que o uso de software nestes contextos pode trazer. Ainda, entende-se que o processo de desenvolvimento de sistemas para tais contextos, torna-se contínuo na medida em que novas necessidades são descobertas e ajustes para eficiência e eficácia tornam-se itens obrigatórios.

Por fim, constata-se que o projeto, desenvolvimento e suporte a um sistema web para tecnologias assistivas requer uma equipe de profissionais que vai além do conhecimento técnico em desenvolvimento de software, linguagens de programação, entre outros. Faz-se necessário o contexto educacional, social e técnico de diferentes áreas, bem como uma aplicação real prática do software em questão, visando à realidade de tais dificuldades/necessidades a serem atendidas. 


\section{. K.'Ingenierías}

\section{REFERÊNCIAS}

Almeida, M \& Bax, M. (2003). Uma visão geral sobre ontologias: pesquisa sobre definições, tipos, aplicações, métodos de avaliação e de construção. Revista Ciência da Informação, 32(3).

Avila, B. G. (2011). Comunicação aumentativa e alternativa para o desenvolvimento da oralidade de pessoas com autismo. Dissertação. Faculdade de Educação. Programa de Pós-Graduação em Educação. UFRGS. Porto Alegre.

Avila, B. G. \& Passerino, L. M. (2011). Comunicação Aumentativa e Alternativa e Autismo: desenvolvendo estratégias por meio do SCALA. In: Anais VI Seminário Nacional de Pesquisa em Educação especial: Práticas Pedagógicas na educação Especial: multiplicidade do atendimento educacional especializado, 1, 1-10.

Bez, M. R. \& Passerino, L. M. (2009). Applying Alternative and Augmentative Communication to an inclusive group. In: WCCE 2009 - Education and Technology for a Better World Monday, 1, 164-174.

Bez, M. R. (2010). Comunicação Aumentativa e Alternativa para sujeitos com Transtornos Globais do Desenvolvimento na promoção da expressão e intencionalidade por meio de Ações Mediadoras. Dissertação. Programa de Pós-Graduação em Educação - Faculdade de Educação. Universidade Federal Do Rio Grande Do Sul. Porto Alegre.

Bez, M. R. (2014). Sistema de comunicação alternativa para processos de inclusão em autismo: uma proposta integrada de desenvolvimento em contextos para aplicações móveis e web. 286 f. Tese (Doutorado em Informática na Educação) - Programa de PósGraduação em Informática na Educação, Centro Interdisciplinar de Novas Tecnologias na Educação, Universidade Federal do Rio Grande do Sul, Porto Alegre.
Berners-Lee, T., Hendler, J. \& Lassila, O. (2001). The Semantic Web. Scientific American, p. 29-37.

Brasil (2008). Política Nacional de Educação Especial na Perspectiva da Educação Inclusiva. Brasília. MEC/Secretaria de Educação Especial 2008. D i s p o nível e m : portal.mec.gov.br/arquivos/pdf/politicaeducespecial. pdf. Acesso em: maio de 2015.

Devmedia (2014). Introdução ao formato JSON. 2014. Disponível e m http://www.devmedia.com.br/introducao-aoformato-json/25275. Acesso em: março de 2016.

Godoy, Arilda S. (1995). Introdução à pesquisa qualitativa. Revista de Administração de Empresas, 35(2), 57-63.

Passerino, L. (2005). Pessoas com autismo em ambientes digitais de aprendizagem: estudo dos processos de interação social e mediação. Tese (Doutorado em Informática na Educação). UFRGS, Programa de Pós-Graduação em Informática na Educação, Porto Alegre.

Passerino, Liliana Maria \& Santarosa, Lucila M Costi (2008). Autism and Digital Learning Environments: processes of interaction and mediation. Computers and Education, 51, p. 385-402.

Passerino, Liliana Maria \& Bez, Maria Rosangela (2013). Building an Alternative Communication System for literacy of children with autism (SCALA) with Context-Centered Design of Usage. Autism / Book 1, 1, 655-679.

Praciano, E. (2014). Os benefícios e as vantagens do P H P. 2014 . D i spon ível e m : $<$ http://elias.praciano.com/2014/02/15-beneficios-evantagens-do-php/>. Acesso em: março de 2016. 


\section{Ingenierías:-.}

Noy, N. F. \& Mcguinness, D. L. (2001). Ontology Development 101: A Guide to Creating Your First Ontology'. Stanford Knowledge Systems Laboratory Technical Report KSL-01-05 and Stanford Medical Informatics Technical Report SMI-2001-0880, March 2001.

Tetzchner, S. \& Martinsen, H. (2000). Introdução à Comunicação Aumentativa e Alternativa. Portugal: Porto.
Vygotsky, L.S. (1998). A Formação Social da Mente. São Paulo: Martins Fontes.

Walter, C. (2011). O PECS adaptado no ensino regular: uma opção de comunicação alternativa para alunos com autismo. In: Nunes, L. Quiterio, P; Walter, C.; Schimer, C.;Braun, P.(Org.) Comunicar é preciso: em busca das melhores práticas na educação do aluno com deficiência. Marilia: ABPEE. 